\title{
Effect of Co catalyst on PECVD growth of carbon nanotubes for NEMS applications
}

\begin{abstract}
In this paper the effect of cobalt (Co) catalyst on the growth of carbon nanotubes (CNTs) was studied. CNTs were vertically grown by plasma enhanced chemical vapor deposition method (PECVD) at $700^{\circ} \mathrm{C}$ with various sputtered Co catalyst thicknesses. Experimental results shows that for carbon nanotube growth duration of 20 minutes, growth was only achieved with thinner catalyst layers but when the growth duration was doubled, high density of CNTS were also observed with thicker catalyst layers with taller nanotubes formed. The nucleation of the catalyst with various thicknesses was also studied as the absorption of the carbon feedstock is dependent on the initial size of the catalyst island.
\end{abstract}

Keyword: Carbon nanotubes; Nanomaterials; Chemical vapor deposition; PECVD; Cobalt; Nucleation 